\title{
Mastopexia tridimensional con anclaje efectivo. Una respuesta a la ptosis, alteraciones de volumen, flacidez y recidiva en las mamoplastias
}

\section{D Mastopexy with a dependable anchoring site. A logical response to ptosis, volume alterations, flaccidity and relapse in mammaplasties}

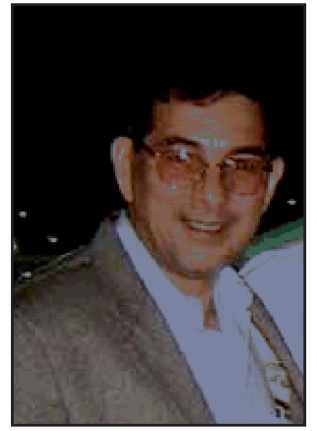

Dr. Guillermo Peña Cabús

Peña Cabús, G. *

Resumen

La mastopexia es la técnica quirúrgica común que la Cirugía Plástica realiza en las mamas. En la literatura, se han expuesto múltiples y muy buenas técnicas sin terminar la búsqueda del elemento que sea consistente y que provea la mayor permanencia de resultados. De manera habitual, la atención se ha enfocado hacia la modificación anatómica del polo inferior, tratando de rellenar el polo superior de la mama de manera indirecta, con la confusión generalmente establecida de que, en la mayoría de las técnicas descritas, se considera el sostenimiento como sinónimo de suspensión.

De manera descriptiva, exponemos una alternativa de suspensión que resulta tan interesante como sencilla, produciendo los resultados esperados a largo plazo y simplificando la técnica al reducir de manera importante la falta de mantenimiento del relleno del polo superior de la mama, el tiempo operatorio y facilitar la reconstrucción del molde mamario. La detección de un punto anatómico de fusión entre la fascia superficial, que es una continuación de la glándula mamaria y la propia fascia pectoral en la pared torácica, de importancia quirúrgica para el anclaje de la glándula mamaria, y el desarrollo de una técnica simple de suspensión glandular, logran cambiar los conceptos acostumbrados de nuestra práctica. La remodelación de la glándula se vuelve más sencilla y se autonomiza el manejo de la cubierta cutánea, permitiendo reducir la longitud final de las cicatrices.

\section{Palabras clave Mastopexia, Suspensión mamaria, Fascia pectoral.}

Código numérico 521-52103

\begin{abstract}
The elusive long term result in mastopexy, reveals the lack of an element that provides with certainty, an alternative that maintains the result surgically obtained. Although myriads of alternatives have been proposed in modern literature, most of them referred to lower pole anatomical molding as an indirect way to modify the upper pole as well, which is in fact the main target expectation for a good and sustained surgically outcome. There is a factor of confusion that has not been currently clarified; sustenance is not necessarily a synonym of suspension, being the latter our main objective that induced to present this technical proposal. The anatomical site of fusion of the superficial fascia which is in continuity with the mammary gland, and the proper pectoralis fascia, means that this structure can be used as a reinforced tissue that becomes as an anchoring site for the breast gland. An easily placement transglandular sutures stablishes the expected real suspension and the technical benefits derived from it, with the advantage of the use of non absorbable suture material that provides the occasion of change for a better good.
\end{abstract}

\begin{tabular}{|ll|}
\hline Key words & $\begin{array}{l}\text { Mastopexy, Mammary suspension, } \\
\text { Pectoral fascia. }\end{array}$ \\
Numeral Code & $521-52103$
\end{tabular}




\section{Introducción}

Los problemas que los cirujanos tenemos con la mastopexia y sus resultados, son congruentes con los múltiples factores involucrados en la misma. La incertidumbre de si la técnica quirúrgica funcionará en cada caso afrontado, nos coloca en una posición que no es ni agradable ni predecible en ciertas situaciones particulares. La avalancha de ideas técnicas expuestas en la literatura, muestra que la diversidad tanto de alternativas quirúrgicas como de acciones resolutivas, se presentan con bastante frecuencia con un enfoque unidireccional, en lugar de con la visión amplia y con la atención multifactorial con que se deben considerar los cambios biológicos que sufre la mama. En esencia, necesitamos una revisión conceptual y una estructuración integral del pensamiento orientadas hacia la mejor resolución técnica del problema. Este artículo pretende ser una propuesta de atención multifactorial y de respuesta hacia una resolución del problema con beneficios consistentes.

\section{Consideraciones anatómicas y técnicas}

La mastopexia es sin lugar a dudas, el procedimiento quirúrgico mamario en Cirugía Plástica que mayor reto anatómico representa cuando se intenta obtener resultados consistentemente satisfactorios y, de forma muy especial, en relación a la permanencia de dichos resultados. Cuando es necesario, tras haber compensado el déficit o el exceso de volumen glandular, es cuando la forma y posición de las mamas se vuelven los factores principales que influirán en el resultado final.

El enfoque tradicional es el de la resección cutánea del polo inferior de la mama que es lo que habitualmente se conoce como mastopexia. Sin lugar a duda, con este método se produce una suspensión y formación de un cono mamario más atractivo, al menos inicialmente; sin embargo, es ampliamente conocido que con frecuencia la suspensión alcanzada se vuelve incompetente debido al peso glandular y/o a la existencia de una cubierta cutánea que no provee el soporte y la resistencia suficientes para evitar el descenso glandular.

Los diferentes diseños de resección cutánea utilizados, representan invariablemente solo eso, resecciones del continente mamario orientadas hacia el tipo de cicatriz resultante, las cuales sin embargo, están sujetas a los cambios gravitacionales producidos en la glándula mamaria, en particular por la relación existente entre su peso y la resistencia cutánea, y que actúan sobre el resultado obtenido quirúrgicamente.

Claramente existen dos dimensiones que se modifican quirúrgicamente $(1,2)$ sobre la pared torácica cuando se realiza una mastopexia: la transversal o de reducción del diámetro de implantación de la glándula y la sagital, por la elevación de la ubicación glandular en relación a la pared torácica. Además, sabemos que la proyección antero-posterior, es decir, la tercera dimensión, se puede modificar mediante la resección de la parte central de la base del cono, posterior al complejo areola pezón, y en el caso de que queramos un aumento mamario, con la inserción de un implante de relleno central

El polo superior vacío, que con frecuencia resulta en la mama femenina a medida que transcurre el tiempo, no es más que la expresión del descenso gravitacional progresivo de la masa mamaria y la falta de mantenimiento de la resistencia por parte de la cubierta externa de la misma. La proyección ántero-posterior se aumenta, al menos temporalmente, con el estrechamiento cutáneo de la base del cono y se reduce con la resección cutánea periareolar. La experiencia nos muestra que la imbricación del tejido glandular del polo inferior con suturas no absorbibles, suele ser suficiente en condiciones particulares de bajo peso glandular y de manera temprana para resolver este problema, pero también puede perder su eficacia con el paso del tiempo. Sin embargo, cuando a pesar de una imbricación adecuada, existe un peso glandular que sobrepasa la resistencia externa, el resultado de la corrección será poco satisfactorio y en muchos casos lo consideraremos y con mucha razón, como una recidiva de la ptosis glandular preoperatoria.

La adecuación externa a ciertos cambios tisulares internos, sin embargo, proporciona una alternativa de mucho interés. La rotura de paradigmas es una postura muy significativa en Cirugía Plástica (3), con el objetivo de buscar la excelencia y resultados anatómicamente satisfactorios. El significado del término quirúrgico mastopexia etimológicamente, es el de fijación del tejido glandular mamario a una alternativa anatómica factible; es decir, a la pared torácica y la técnica está orientada a reducir las recidivas, especialmente en condiciones de igualdad o sobrepeso glandular (o también el creado por el peso añadido al colocar implantes mamarios). La experiencia personal obtenida al encontrar una estructura anatómica que proporciona un sitio preciso de anclaje y el desarrollo adicional de una técnica quirúrgica que provee, mediante una sutura transglandular, la suspensión mamaria necesaria para tratar una gran variedad de condiciones que se nos pueden presentar, son las razones que nos han motivado a compartir y divulgar una alternativa quirúrgica bastante práctica, que resulta atractiva por eficiente además de por ser muy fácilmente reproducible.

En la pared torácica anterior existe un sitio anatómico constante, que aunque no se ha considerado habitualmente como tal, en realidad ha demostrado ser de gran importancia quirúrgica, hasta donde hemos podido comprobar. En nuestra experiencia, encontramos una idea novel que luego dio lugar a establecer una técnica protocolizada de utilidad práctica. La estructura anatómica a la que hacemos referencia se utiliza en esta técnica como punto de anclaje, mediante sutura no absorbible, para la masa glandular mamaria (Fig. 1 A,B). La suspensión que se produce reduce la magnitud de las maniobras que de 

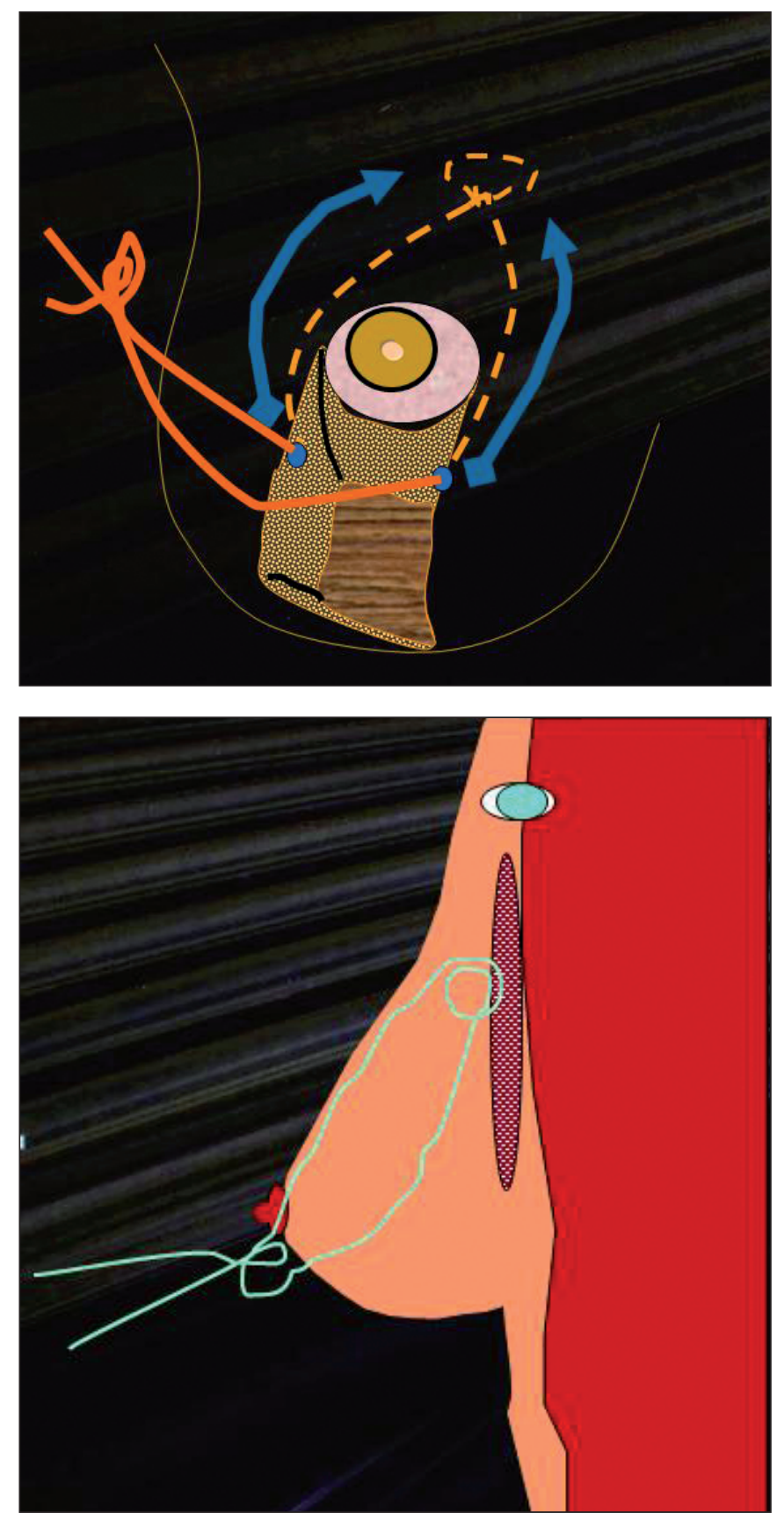

Fig. 1. Esquema de la colocación de la suspensión a la pared torácica. A) vista anteroposterior. B) Vista lateral

otra manera serían necesarias para la remodelación mamaria. El sitio en cuestión, se determina en la fusión de la fascia pectoral y la fascia superficial que ocurre de manera constante alrededor de 5-6 cm caudalmente a la clavícula y en el eje mamario. La estructura fascial encontrada es lo suficientemente rígida por su engrosamiento a nivel de la unión y se localiza entre la $2^{\mathrm{a}}$ y $3^{\mathrm{a}}$ costillas sobre la pared anterior del tórax.

Tanto en disecciones en cadáver como en casos clínicos, encontramos que este punto de reforzamiento fascial se presenta de manera constante, simétrica y suficientemente resistente como para ser un sitio de anclaje quirúrgico. No de menor importancia es el hecho de que este punto se encuentra en posición cefálica y por fuera de la glándula mamaria, por lo que se obvia la po- sibilidad de interferir con las rutas linfáticas mamarias de importancia clínica, especialmente desde el punto de vista oncológico.

Durante más de una década hemos desarrollado gradualmente la técnica introduciendo múltiples cambios hasta conseguir una rutina quirúrgica estable para proveer anclaje a la glándula mamaria, reducir el desplazamiento caudal consecuente al peso y compensar la falta de soporte externo, elementos críticos que frecuentemente condicionan nuestros resultados.

\section{Técnica quirúrgica}

Marcaje y Abordaje. Utilizamos las estructuras anatómicas fijas del tórax para marcar las longitudes existentes en las mamas y así lograr una apreciación realista y muy objetiva de lo existente y del posible cambio a conseguir quirúrgicamente. Consideramos que la piel mamaria, especialmente en condiciones de hipertrofia y flacidez marcadas, se convierte en un factor poco confiable de marcaje sobre la misma mama, lo cual se puede apreciar fácilmente por las variaciones experimentadas con los cambios posturales. En cambio, el marcaje empleado en la técnica que describimos se limita casi en exclusiva a: a) la línea de dirección del eje mamario, b) el surco submamario, c) la posición del pezón sobre la línea media, que se puede anticipar, pero que preferentemente se marca al terminar el primer lado operatorio. La resección glandular, si fuera necesaria, se marca de forma convencional orientada sobre la continuación dibujada del eje mamario caudal al complejo areola pezón (CAP). En la mayoría de las intervenciones, después de una prudente actitud en el primer lado, se trata de reproducir lo obtenido en el segundo lado. Sin embargo, consideramos que la decisión de realizar o no marcas preoperatorias en las mamas es exclusiva de cada cirujano, sin necesidad de objetársele la preferencia adoptada.

De manera rutinaria, realizamos la intervención bajo sedación intravenosa y anestesia infiltrativa con cánulas finas de punta roma y orificios laterales; de esta manera conseguimos una reducción importante de la pérdida sanguínea y de las molestias postoperatorias. Tanto en mastopexia con o sin implantes, el abordaje se hace a través de una incisión oblicua y de preferencia curva en sentido lateral, con resección cutánea conservadora, por debajo del CAP, hasta un punto 2-3 cm por encima del surco submamario, para facilitar la compensación final de la cubierta cutánea. La resección final de piel y la reubicación del CAP, que se vuelven de esta manera mucho más sencillas, se realizan después de la remodelación del parénquima glandular, ajustando la cubierta de acuerdo a la necesidad existente y a las condiciones preferentes, ofreciendo la posibilidad de obtener una menor longitud de la cicatriz final resultante.

Cuando optamos por una reducción glandular, el axioma de uso habitual se limita a que no se trata de 
cuánto peso se reseca, sino que lo que importa es cómo queda el remanente glandular que se ha dejado para cada situación individual. Habitualmente, la resección se hace en el polo inferior y siempre caudal al meridiano neurovascular descrito por Wuringer (4) y la resección se orienta sobre el eje mamario para mejorar el cuadrante ínferoexterno, que habitualmente es la parte de mayor volumen en exceso. Si se requiere resección adicional de tejido, se logra retirando volumen primero de los pilares glandulares, luego de la base central y finalmente de la porción de glándula que conforma el surco submamario, separando la glándula de la piel y del celular subcutáneo, evitando resección de piel mamaria que puede convertirse fácilmente en cubierta torácica sin necesidad de ser resecada y evitando así crear una cicatriz permanente y de ubicación muy notoria.

Si se decide colocar un implante de relleno central, lo haremos mediante la técnica transpectoral superior o doble plano $(5,6)$, por las ventajas que ofrecen, para evitar la deformidad producida por el inconveniente desplazamiento cefálico de los implantes y las deformidades tipo bandas, indentaciones o cambios de forma, debidas a la reinserción postoperatoria del músculo pectoral en la glándula mamaria, que es la causa real de las alteraciones morfológicas mencionadas.

Es en este momento, cuando las tres alternativas de cirugía mamaria coinciden en una alternativa técnica común: las tres suelen requerir de una suspensión interna y su subsecuente remodelación para simplificar los detalles técnicos.

La disección para encontrar el sitio de anclaje se realiza hasta la parte más cefálica del tórax anterior, lo que permite la disección digital en el plano prepectoral siempre orientada en la línea del eje mamario; se trata de una maniobra sencilla, ya que no se puede pasar fácilmente mas allá del sitio de fusión facial. De esta manera, la disección en sentido cefálico es limitada pero suficiente para colocar la sutura de suspensión. Al mismo tiempo, existe menos riesgo de interferir con el plano de los linfáticos de la mama y no existen estructuras neurovasculares importantes. Llegamos así fácilmente al punto de límite superior de la disección, que está en la fusión de ambos planos fasciales y se localiza entre la $2^{\mathrm{a}}$ y $3^{\mathrm{a}}$ costillas. A partir de ese momento, hacemos prueba de tracción tomando tejido con una pinza de Allis hasta encontrar el nivel mas superior posible que produzca la menor indentación visible en el exterior del tórax. Luego colocamos a ese nivel el punto primario de anclaje con Polipropileno 0, abarcando la fascia pectoral de la forma más amplia posible y comprobamos su eficiencia traccionando de él. Posteriormente anudamos asegurando que no se causa isquemia para mantener indemne el sitio de suspensión y luego pasamos cada uno de los dos extremos de la sutura transglandular (a $2-3 \mathrm{~cm}$ de profundidad del tejido mamario para evitar indentaciones en la superficie), produciendo el efecto deseado de suspensión
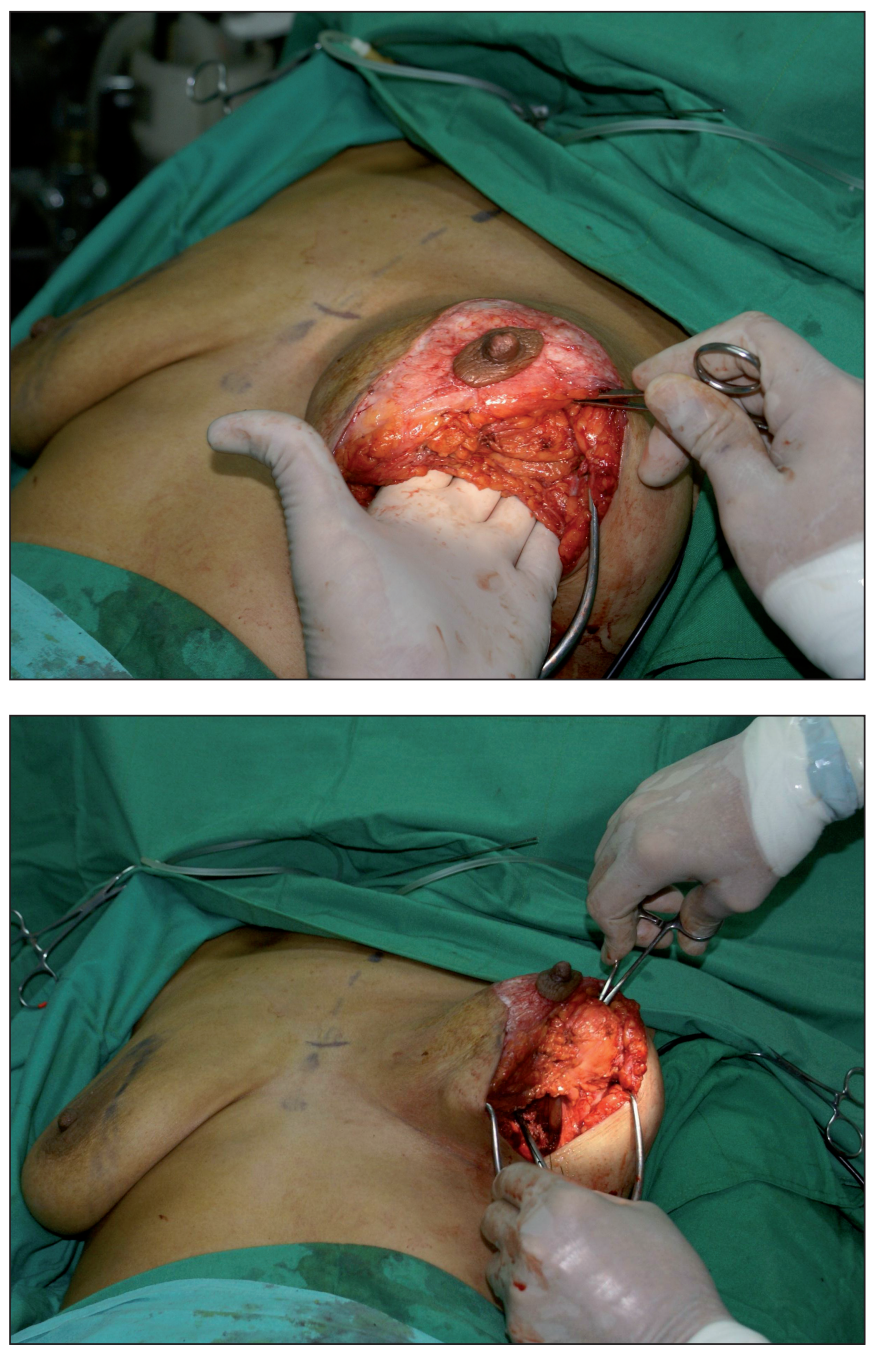

Fig. 2. Secuencia del trayecto transglandular de la suspensión, pasando un extremo de la sutura de suspensión hacia cada pilar.

A) Manera de pasar una pinza hemostática con un trayecto curvo, profundizando hasta llegar lo más cerca posible del sitio de anclaje. B) Se toma uno de los extremos y se regresa sobre el trayecto.

cercano al CAP (Fig. 2A,B). Con una pinza hemostática larga y curva, iniciamos la ruta curva con convexidad hacia fuera con referencia al lado del pilar mamario en que se entró y la dirigimos en sentido craneal y hacia la cara posterior de la glándula, para egresarla lo más cerca posible del punto de anclaje sobre el pectoral. Tomamos el extremo de la sutura con la pinza y se retrae hasta su sitio de entrada.

Ambas suturas siguen una trayectoria curva para aglomerar una cantidad suficiente de tejido mamario en el polo superior, se retiran a través de los pilares, calculando egresar a 2-3 cm caudalmente con respecto al CAP, y se mantienen sin anudar por completo, acortando la longitud del polo superior y empujando la glándula en sentido craneal, hasta alcanzar la mayor elevación deseada. En este momento colocamos una segunda sutura de suspensión que toma los dos pilares de la glándula, pero caudal con respecto a la anterior (sutura primaria) y la anclamos a la fascia pectoral superior. Con esto, distribuimos el peso entre las dos suspensiones, haciéndolas más eficientes y compartiendo la distribución de la suspensión. En las mamo- 

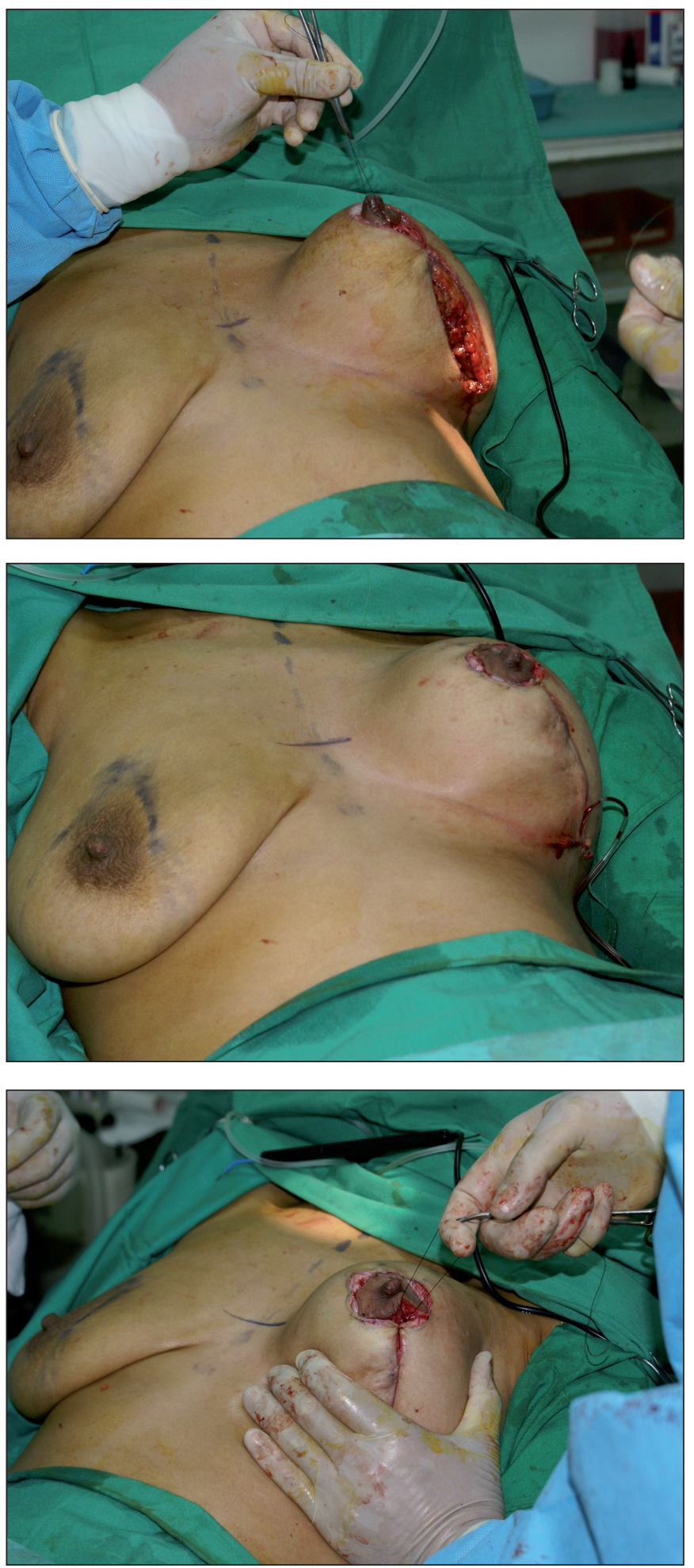

Fig. 3. Con anudado incompleto y sostenida de manera firme en alto: A) Se coloca la segunda sutura de anclaje uniendo los dos pilares de la glándula a la fascia pectoral superior, reconstruyendo gradualmente el defecto glandular de la resección. B) Se completa el cierre cutáneo C) Se ajusta la glándula a la altura que corresponde, levitándola con una mano y ajustando la sutura de suspensión primaria, y se completa el nudo de la sutura.

plastias de reducción, con frecuencia es necesario medializar ambas mamas cuando se presentan con movilidad excesiva y se lateralizan cuando la paciente está en posición supina; en estos casos resulta beneficioso colocar una tercera sutura anclada medialmente. Con esto, observamos que se completan los puntos necesarios de suspensión.
Por la indudable existencia de contaminación endógena (7) de la propia glándula mamaria, aplicamos solución antiséptica de polivinilpirrolidona yodo durante unos segundos y luego irrigamos y lavamos con solución salina normal y antibiótico profiláctico. En el caso de uso de implantes para relleno central, estabilizamos su posición mediante la colocación de suturas no absorbibles a la pared torácica, de manera que logramos la creación de un zócalo subfascial (8).

$\mathrm{Al}$ suspender la sutura primaria (Fig. $3 \mathrm{~A}-\mathrm{C}$ ) podemos reconstruir la glándula existente con una mayor facilidad. Esta sutura primaria provoca la elevación y giro del CAP en el cono mamario, reduciendo el tamaño del área de desepitelización necesaria para su reubicación de manera anatómicamente más agradable y también reduciendo la tensión sobre la línea de cierre periareolar, factor muy deseable para reducir la posibilidad de cicatrización hipertrófica.

Cierre cutáneo. La otra variante técnica beneficiosa que introducimos con la técnica descrita ha sido el cierre de la cubierta cutánea (Fig. 4 A-D). La baja tolerancia por parte de las pacientes a las cicatrices extensas después de la cirugía mamaria, ha conducido invariable-
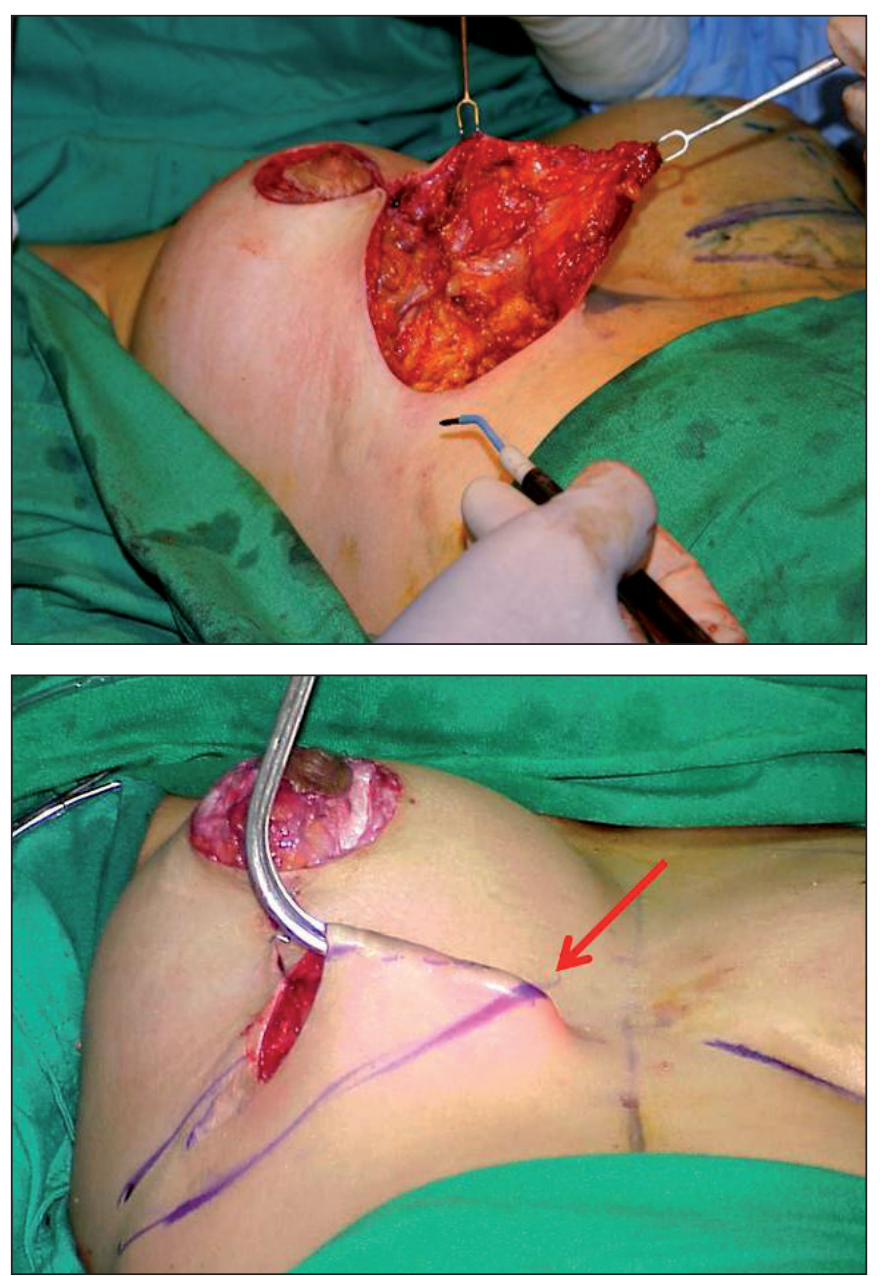

Fig. 4. Maniobras y opciones de cierre cutáneo para reducción de la cicatriz final.

A) Elevación de un colgajo subcutáneo medial para lateralizar el cierre y hacerlo menos notorio B) Resección glandular caudal si es necesario, para crear un nuevo surco submamario mas elevado y reducir la distancia final hasta el CAP, además de convertir piel mamaria en piel de pared torácica. 

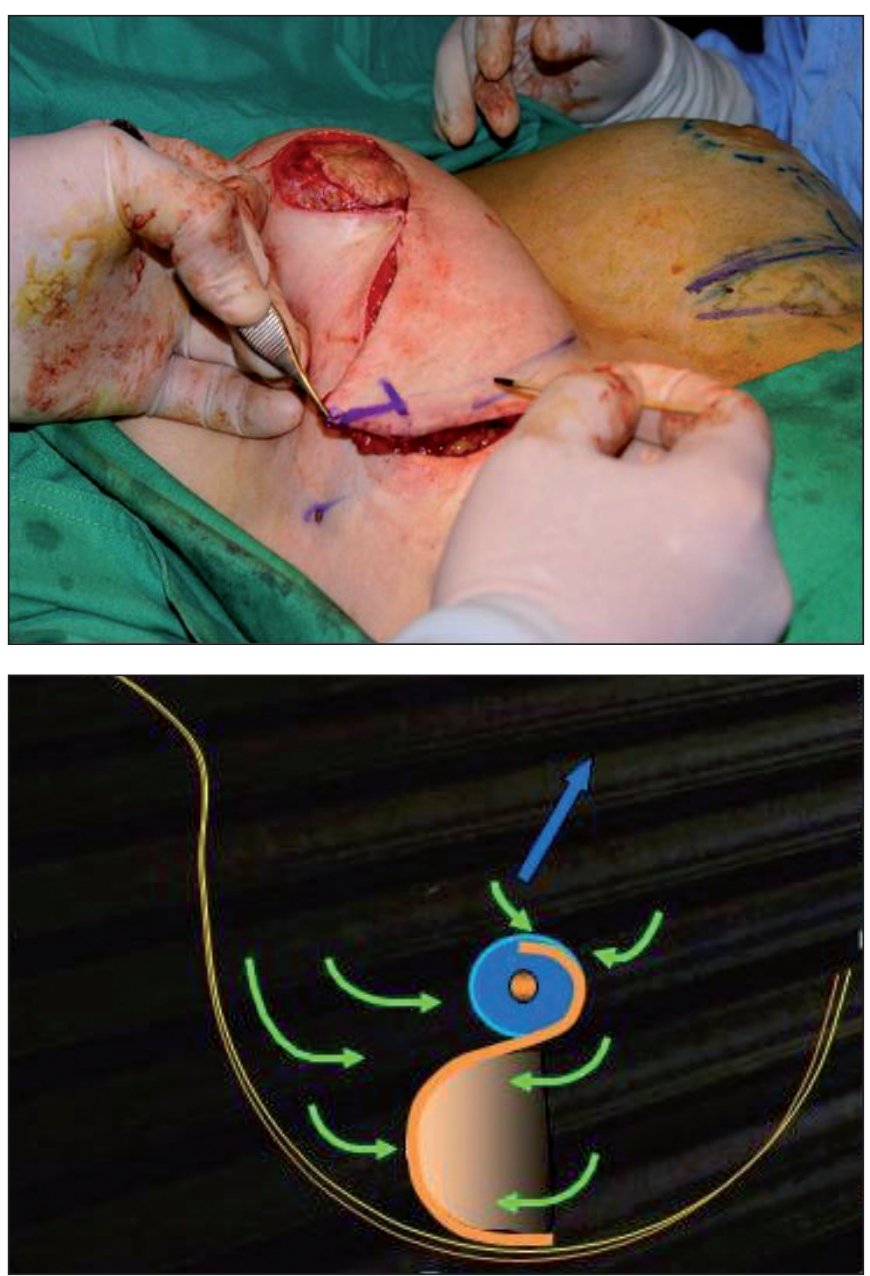

Fig. 4. C) Al autonomizarse el cierre cutáneo, se redistribuye la piel hacia la línea de cierre más favorable y corta posible. D) El trazo de cierre preferido es de tipo curvo, como se muestra en el esquema, aunque si es preciso se puede convertir en una T pequeña y lateral a la línea media de la mama.

mente a la búsqueda de una reducción de la longitud de las mismas. Después de obtener la suspensión efectiva y la remodelación glandular, el cierre cutáneo se vuelve un elemento operatorio autónomo, pudiendo modificar sus trazos a voluntad y obviar así la longitud mayor de las cicatrices, acostumbrada en muchas de las técnicas quirúrgicas habitualmente empleadas cuando se piensa que existe un exceso cutáneo importante. El hecho de cambiar a una ubicación más oblicua las incisiones de buena calidad, hace que las cicatrices resultantes sean aun menos aparentes en la vista de frente; el otro beneficio logrado es el de reducir la incisión a lo largo del surco submamario, que tan visible resulta en posición supina y que en posición erecta solo se oculta cuando resulta una ptosis postoperatoria. La redistribución de piel que logramos con nuestra técnica, resulta en la mayoría de casos en una cicatriz de menor longitud de lo aparentemente necesario. Sabemos que los trazos curvos de cierre cutáneo consumen una mayor cantidad de piel que los trazos rectos combinados, por lo que su empleo resulta entonces apropiado en una anatomía con esas características particulares. El ajuste de volumen antes del cierre subdér-
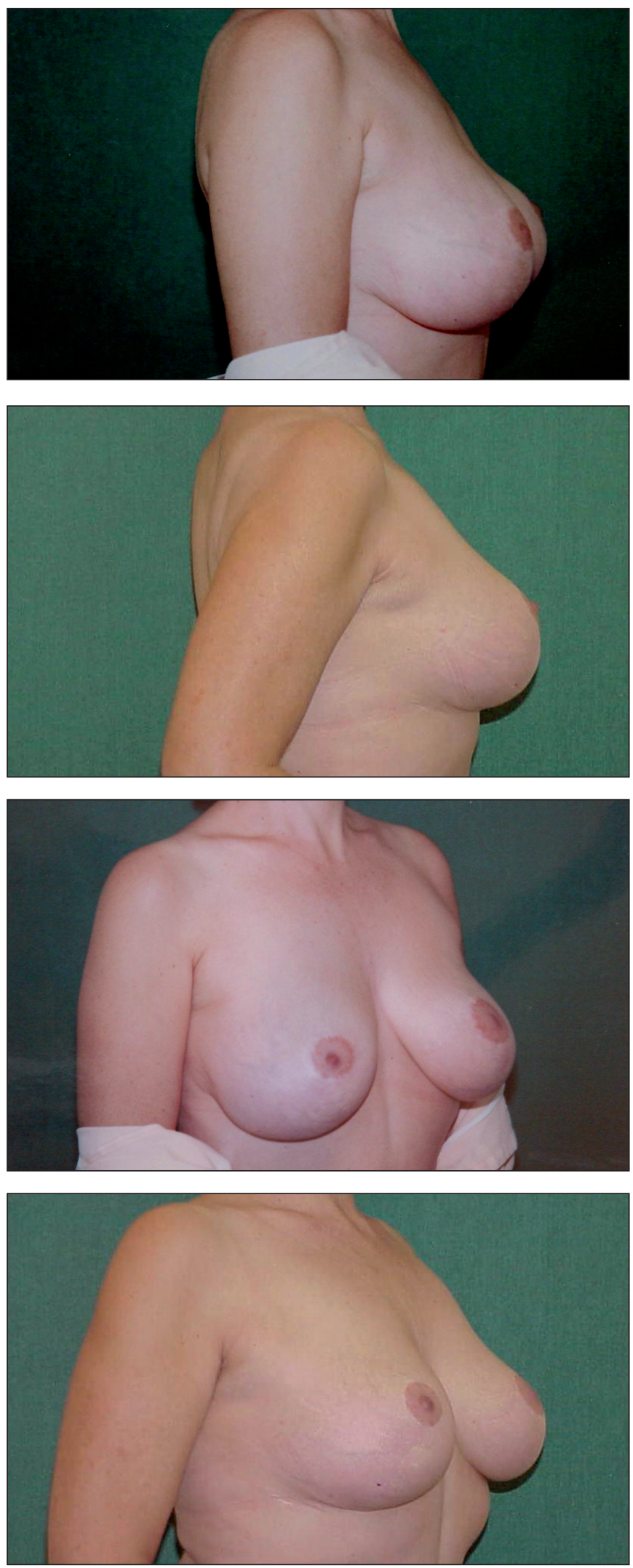

Fig. 5. Mujer de 40 años multípara, a la que ya le habían realizado una reducción glandular previa en la adolescencia. Vistas pre y postoperatoria a los 9 años de la intervención. A) y B) Vista lateral, C)y D) Vista oblicua.

mico, en realidad simplifica y acorta el tiempo transoperatorio. Cuando se considera necesario, se puede recurrir a una compensación, resultando una incisión en $\mathrm{T}$ pequeña sobre el surco submamario (Fig. 5 A-D). 
Drenajes. La extracción de fluidos es solo una parte del drenaje. Sin importar el calibre empleado, un drenaje no detiene un hematoma en formación, especialmente si se forma rápidamente un volumen considerable, pero sí permite que sea evidente de manera más temprana que cuando ya es clínicamente obvio. La condición de lo que acontece internamente se manifiesta también de forma temprana por el contenido del drenaje, y por supuesto, es también una forma de obtener una muestra objetiva para ser examinada en el laboratorio. Pero la aplicación más reciente ha sido la de poder introducir anestésicos de duración prolongada para reducir las molestias postoperatorias de manera fácil y rápida, logrando el control del dolor que se busca y reduciendo considerablemente el requerimiento de analgésicos.

\section{Discusión}

El establecer una rutina con sistematización tanto lógica como efectiva y con resultados consistentes en Cirugía, es una posición a tener en cuenta en los conceptos modernos de seguridad operatoria, lo cual es una condición imperante para beneficio del paciente, aunque por si misma cause controversia importante o se aleje de la formalidad instituida como modo de actuar habitual. El romper paradigmas establecidos y llevarlos a condiciones intuitivas y lógicas ha sido la base de nuestra especialidad (1-3). Los cambios introducidos permiten hacer más atractiva nuestra práctica, siempre y cuando se orienten hacia el beneficio bien entregado. Creemos que la buena experiencia obtenida debe ser compartida, descriptivamente al menos de forma inicial, pero con la seriedad científica que corresponde. Posteriormente continuará con la comparación requerida para validar su eficacia y medir el impacto de cambio y beneficio obtenido.

Los cambios técnicos aquí descritos, se han empleado en los casos operados en un período de 13 años, de manera individual cumpliendo con los criterios de inclusión, hasta constituirse en una rutina sistematizada que simplificó el enfoque de la técnica operatoria tradicional. En breve se enviará la revisión y análisis del material clínico producido en los 13 años de su empleo.

Encontrar como suspender el tejido en la cirugía de la mama, particularmente si ésta es pesada (Fig. 6 A-D, 7 A-D), convierte lo usual en una condición intuitiva y largamente buscada. Las mamas se suspenden desde un punto anatómico que debe ser fácilmente identificable, que lo permita, y que prevenga el que la glándula cuelgue por sus condiciones y sin control de una pared torácica que no cuenta con un elemento de enlace que lo evite. El solo hecho de encontrar una estructura anatómica de importancia quirúrgica y comprobar de manera constantemente auténtica su utilidad, facilitando la práctica personal, creo que vale la pena de ser compartido.

La fusión de la fascia pectoral con la fascia superficial torácica, es el punto más cefálico sobre la pared del tórax
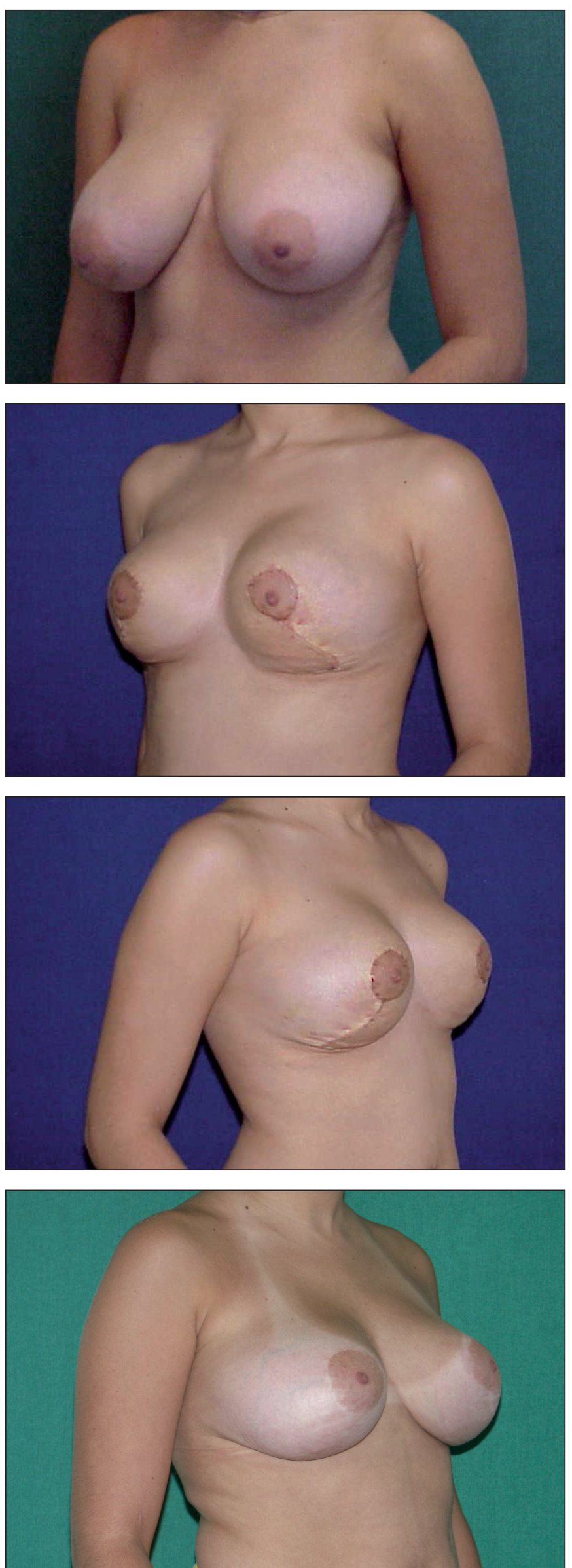

Fig. 6. Mujer de 24 años, nulípara, que prefería conservar un volumen importante. A) Imagen preoperatoria. B) y C) Imagen a las 3 semanas de la cirugía. D) Imagen a los 3 años de postoperatorio; a pesar del aumento de peso, mantiene bastante bien el cambio temprano logrado. 

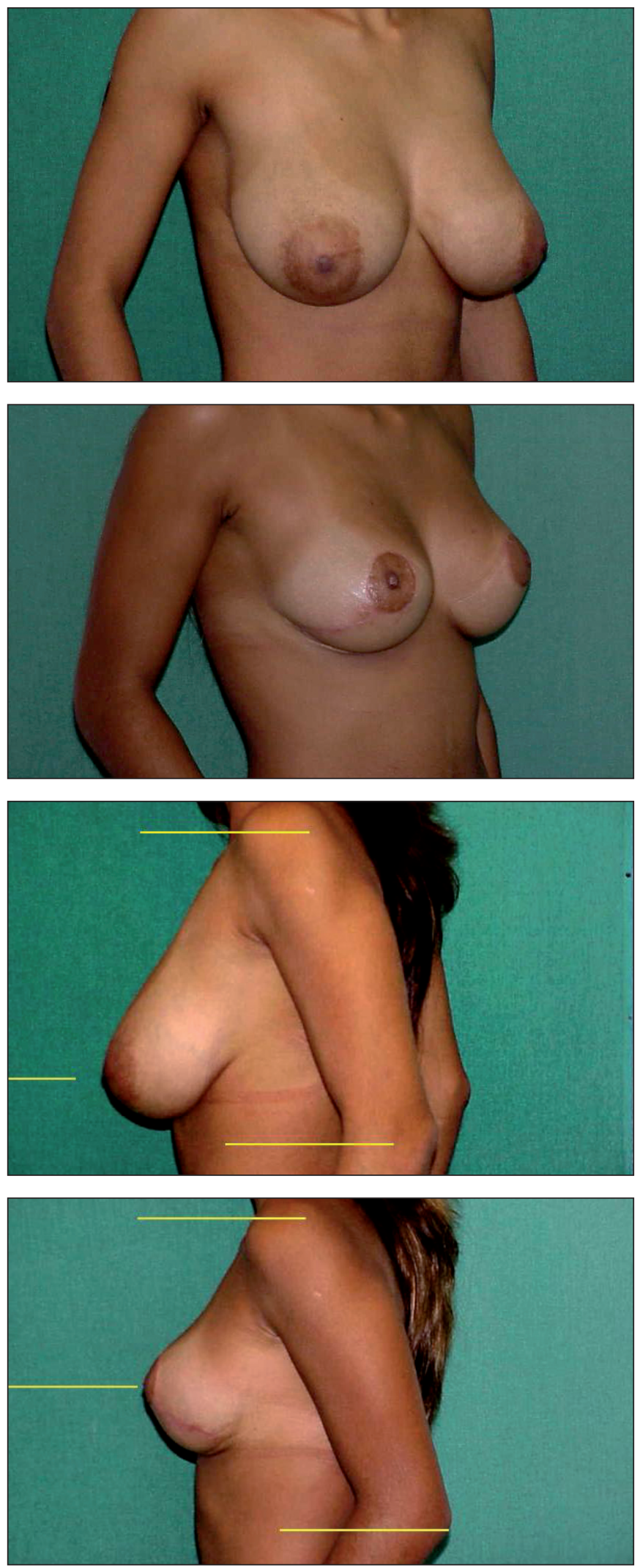

Fig. 7. Mujer de 24 años, nulípara, con un importante peso glandular sintomático. Imágenes pre y postoperatorias. A) y B) Vistas oblicuas. C) y D) Vistas laterales.

hasta el que se permite la disección roma sobre el plano pectoral. Esta estructura permite crear un punto de suspensión suficiente y eficiente para el tejido mamario que va a ser modificado quirúrgicamente. La reposición y mantenimiento del volumen glandular en el polo superior constituye uno de los elementos más destacados en cuanto al resultado a obtener (8 A-D). Asimismo, la suspensión glandular con la sutura secundaria, incrementa la proyección anteroposterior de la mama, y ésta es la tercera dimensión a conseguir.
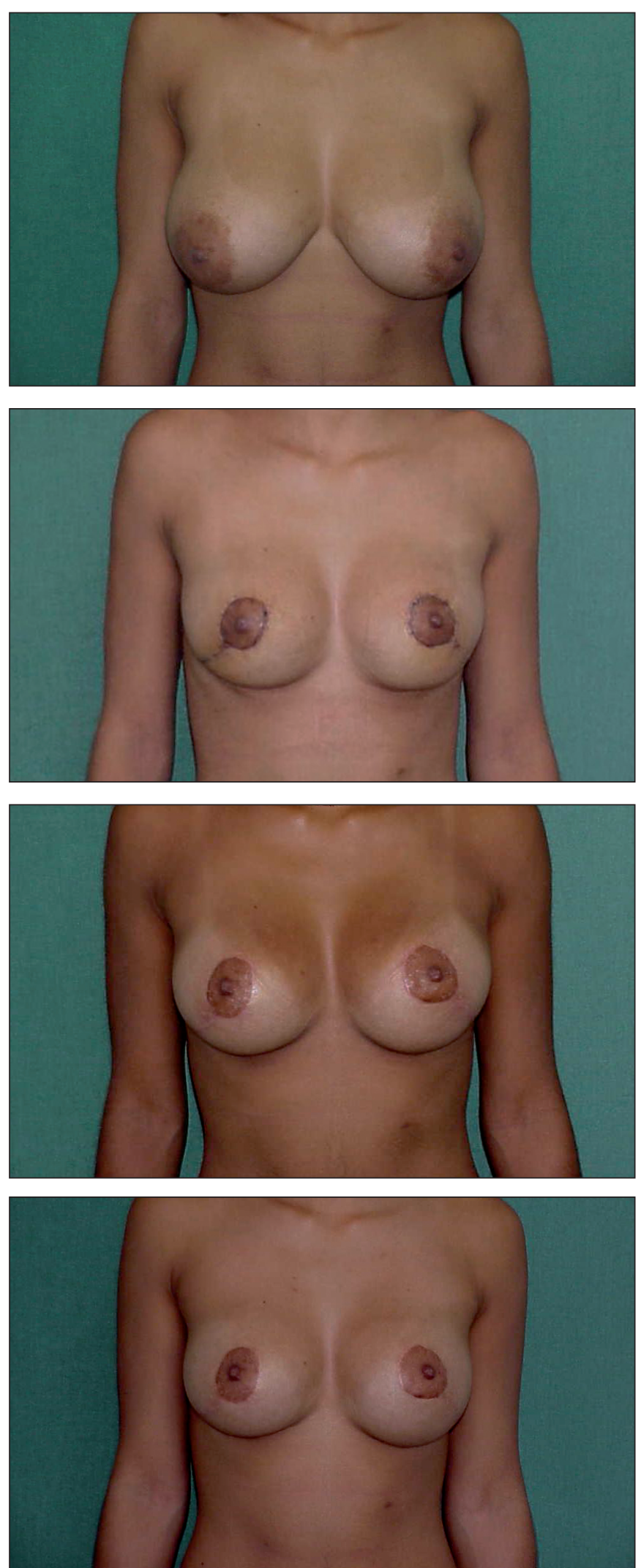

Fig. 8. Mismo caso de Fig. 7, en la vista anterior.

A) Preoperatorio, B) Imagen a los 15 días de la resección y suspensión interna, C) Imagen a los 6 meses de postoperatorio, D) Imagen a los 18 meses de postoperatorio. Las medidas postoperatorias se mantienen de manera bastante similar, mejorando la forma después de los 6 meses de postoperatorio, aun con aumento de peso. 
De manera esperada, existe un grupo menor de pacientes que muestran relajación de las distancias inicialmente obtenidas, lo que puede ser atribuido a razones técnicas o también a la particular constitución del parénquima mamario. Sin embargo, la vasta mayoría de pacientes, refiere satisfacción con el mantenimiento del resultado.

El hecho de insertar un sencillo detalle técnico que al final permite una reducción de tiempo operatorio y facilita la reconstrucción del molde mamario, creemos que por si solo tiene mérito. La necesidad de una menor área de desepitelización para colocar el CAP en el vértice del cono mamario y la redistribución de la cubierta según sea necesario, también puede conducir a una simplificación técnica de beneficio

\section{Conclusiones}

Describimos un enfoque técnico que, ejecutado de manera protocolizada, permite simplificar el reposicionamiento quirúrgico de las mamas, con un singular bajo impacto de alteración anatómica o funcional. El cambio técnico sugerido, se limita a una propuesta de maniobras sencillas y fáciles, que acortan el tiempo operatorio. En realidad no es un solo cambio técnico, sino una sumatoria de cambios intuitivos y lógicamente estructurados, orientados hacia la simplificación de procedimientos en cirugía mamaria. La sencillez del marcaje sobre estructuras fijas del tórax, proporciona una objetividad anatómica independiente de la calidad del tejido mamario a tratar y de los cambios posturales de la paciente. Además, presentamos los beneficios de crear un anclaje del tejido glandular a un punto anatómicamente constante, eficiente y confiable, que facilita la remodelación del parénquima mamario y vuelve autónoma la redistribución de la cubierta cutánea, orientada hacia una cicatriz final menos notoria.

Gradualmente hemos ido introduciendo diversas alternativas técnicas, que nos han llevado a conformar lo que ahora es una rutina mucho más aplicable de manera común que por situación particular. El proceso tomó mucho tiempo hasta lograr la combinación de beneficio entre la satisfacción de la paciente y la bona praxis médica involucrada.

\section{Dirección del autor}

Dr. Guillermo Peña Cabús

Centro de Cirugía Plástica

23 Ave S.O. . $3^{\text {a }}$ Calle.

San Pedro Sula, Honduras

e-mail: gpcbiomat@plasticsurgeryhn.com

Bibliografía

1. Marchac D., De Olarte G.: "Reduction mammaplasty and correction of ptosis with a short inframammary scar." Plast. Reconstr. Surg. 1982; 69: 45.

2. Erol O., Spira M.A.: "A mastopexy technique for mild to moderate ptosis". Plast. Reconstr. Surg. 1980; 60(3): 497.

3. Hudson D.A.: "A paradigm shift for Plastic Surgeons: No longer focusing in excising skin excess. Plast. Reconstr. Surg. 2000; 106(2): 496.

4. Wuringer E., Mader N., Posh E., Holle J.: "Nerve and vessel supplying ligamentous suspension of the mammary gland." Plast. Reconstr. Surg. 1998; 101:1486.

5. Peña Cabús G.: "Colocación transpectoral de implantes mamarios. Relevancia técnica y anatómica". Cir.plást. iberolatinoam. 2008; 34(4):259.

6. Ventura O.D., Roseti R.E., Marino H., Marcello G.: "Mamoplastia de aumento en doble plano: una alternativa subfascial en la optimización de las indicaciones". Cir. plást. iberolatinoam. 2008; 34(4): 249.

7. Peña Cabús G.: "Influencia de las cargas triboelectricas y de la contaminación de implantes." Cir. plást. iberolatinoam. 2007; 33(4): 209.

8. Ventura O., Marino H.,Marcello G., et al.: "Un zócalo como soporte del implante mamario." Cir. plást. iberolatinoam. 2007; 33 (1):31 


\title{
Comentario al trabajo: "Mastopexia tridimensional con anclaje efectivo. Una respuesta a la ptosis, alteraciones de volumen, flacidez y recidiva en las mamoplastias"
}

\author{
Dr. Guillermo Vázquez, Buenos Aires (Argentina) \\ Director del Comité de Eventos Internacionales FILACP 2008-2012. \\ Director del Capítulo de Estética de FILACP 2006-2008. \\ Director del Capítulo de Mama de FILACP 2002-2006.
}

El Dr. Guillermo Peña Cabús nos presenta una alternativa de suspensión para evitar las recidivas en las Mastopexias. En la actualidad la mayoría de la técnicas fijan los colgajos, sean a pedículo superior o inferior, a la fascia anterior del pectoral mayor tratando de evitar la recidiva.

La unión de la fascia superficial y la fascia anterior del pectoral mayor es un punto bien definido y es, anatómicamente, donde comienza la región mamaria en su punto superior y como bien dice el autor, se sitúa a entre 5 y $7 \mathrm{~cm}$. aproximadamente del reborde clavicular inferior, dependiendo del tamaño del tórax de la paciente.

Las causas de la ptosis mamaria pueden ser variadas (hipotrofia postlactancia donde el tejido glandular es escaso, ptosis e hipertrofia mamaria asociada, aumento y descenso brusco de peso, etc.); por ende, cada una tendrá el tratamiento adecuado a su etiología.

Los casos que nos muestra el Dr. Peña Cabús son más asociados a hipertrofias que a ptosis puras con poco tejido glandular. En la Fig. 5 no apreciamos la toma de frente, siendo esta posición fotográfica importante e interpreto que la paciente fue operada anteriormente ya que se visualizan cicatrices periareolares. Sería interesante conocer la casuística, edad de los pacientes, el tiempo de evolución de los postopertorios, complicaciones, etc, ya que no figuran en el texto.

En el desarrollo del trabajo manifiesta que esta técnica puede utilizarse también con implantes mamarios. Como este procedimiento rellena el polo superior, considero que una buena alternativa sería usar implantes anatómicos.
Muchos autores han considerado la suspensión como algo importante en la cirugía mamaria. Joao Sampaio Goes de Brasil, utiliza una malla de silicona como un brazier o sujetador interno que le permite modelar la glándula. Luego Ricardo Bustos aplicó este concepto mediante la maya con cubierta de poliuretano.

Coincido con el autor en que la marcación de la piel en una flacidez acusada no es tan confiable y es probable que necesite ajustes durante la cirugía. En cambio en la hipertrofia es más predecible.

Un punto importante es la tensión que se le aplique a esa suspensión, ya que si es excesiva puede conificar excesivamente la mama y por ende tener un sobrante de piel en el surco submamario con una cicatriz resultante mayor. También sería posible colocar más de un punto de suspensión para evitar la posibilidad de que éste se desgarre por alguna maniobra postoperatoria.

Creo que el concepto del Dr. Peña Cabús es distinto a los paradigmas actuales y como él bien dice: "El proceso tomó mucho tiempo hasta lograr la combinación de beneficio entre la satisfacción de la paciente y la bona praxis médica involucrada".

Todas las técnicas conocidas están descritas y publicadas, pero los resultados dependen de quienes las ejecutan.

Felicito al autor por la constante intuición e inquietud para mejorar los resultados a base de procedimientos sencillos, los cuales deberán ser corroborados con un seguimiento a largo plazo. 


\section{Respuesta al comentario del Dr. Guillermo Vázquez}

\section{Dr. Guillermo Peña Cabús}

La dinámica de opiniones ciertamente establece la importancia de objetar o en su defecto apuntalar, lo determinante de las publicaciones científicas de nuestra Revista. El Dr. Vázquez, con su reconocida experiencia en Cirugía Mamaria representa un bastión de opinión, por lo que en verdad agradezco su participación ya que aporta, y aun más oportunamente amplía, mis expectativas personales a la comunicación que intento compartir; además, me incita a una respuesta congruente a su altura profesional. En esta ocasión el escrito presentado se concreta a una comunicación descriptiva, preliminar, de una experiencia interesante de ser tomada en cuenta para un análisis comparativo ulterior.

Conocemos, y resulta muy aceptable, que el estudio de la Anatomía Humana tiene pocos pero si algunos cambios significativos de interés clínico y quirúrgico, identificables como nuevos después de tanto escrutinio ya conocido. Los Cirujanos Plásticos, técnicamente solo nos servimos de ella (la Anatomía) para justificar nuestros propósitos de cambios morfológicos en beneficio del paciente; eso debe llamarse también oportunidad. La estructura mencionada en el artículo, es decir, la fusión fascial entre la fascia superficial y la pectoral, se convierte en un punto de interés clínico cuando se utiliza como un sitio de anclaje quirúrgico nuevo, es decir, la estructura siempre ha estado presente, pero sin embargo no se le ha encontrado la justificación de uso en lo conocido y planteado, especialmente después de la búsqueda realizada en la literatura que se logró revisar. Creo sin embargo conveniente aclarar el concepto principal. Una glándula muy pesada se cae más fácilmente que una que no pesa más allá del soporte cutáneo intencionado quirúrgicamente. Lo opuesto a una mama muy pesada, por supuesto se resuelve con procedimientos de menor magnitud técnica mediante la reducción del continente en su polo inferior. Pero si en el artículo se muestran casos de mamas hipertróficas y pesadas, es porque éste fue el único objetivo a demostrar y buscado, en un intento por suspenderlas de manera efectiva, así como por evitar la ptosis esperada con las técnicas acostumbradas. Las glándulas mamarias que no pesan lo suficiente para causar una ptosis, merecen una diferente consideración técnica, por supuesto diferente también a la conceptualización presentada. En cuanto a utilizar más de una sutura de suspensión, en efecto es una opción muy acertada y así se describe en el texto. La suspensión y remodelación glandular, en efecto, es distribuida en 2-3 suturas entre glándula y pared torácica.

El intentar romper un paradigma no es nuevo en nuestra especialidad, pero es muy difícil de aceptar como tal en un principio, a pesar de ser ampliamente expuesto y de manera fundamentada. El utilizar como alternativa diferente marcajes fijos en la pared torácica con relación a un tejido blando por excelencia, que cambia con la posición expuesta, se presenta como factible. Resulta además muy difícil de aceptar que una débil cubierta cutánea pueda sostener un cierto peso que so- brepasa su propia capacidad de retención. En este punto, lo expuesto ahora se vuelve una respuesta técnica de beneficio. En cuanto a las fotografías del caso 5, como se menciona, fue una reducción-mastopexia secundaria que había sido operada en otro país previamente y se muestra la fotografía de 9 años de seguimiento y con una edad mayor de 50 años. Además considero mejor ejemplificación y de importancia mayor de apreciación en mastopexia la fotografías en posición de tres cuartos y laterales, más que en la frontal, en cuanto a la valoración de la suspensión quirúrgica obtenida y mantenida.

Con referencia a la similitud de las técnicas acotadas, es bueno recordar que las comunicaciones muy bien conocidas de Sampaio Goes y Bustos representaron alternativas de novedad con los nuevos biomateriales disponibles al tiempo de las publicaciones. Ambos biomateriales (cuestionables posteriormente, por cierto), fueron colocados de manera fácilmente identificable en posición supraglandular, con una disección extensa obligada, a manera de suspensión alternativa, pero sin embargo nunca fueron colocados transglandulares, como aquí en este artículo se presenta. La primera opción, reduce invariablemente la proyección ántero-posterior en aras de una suspensión; la segunda opción, aumenta la proyección antero-posterior al conificar con una banda alrededor de la periferia de la glándula mamaria sobre la pared torácica. Adicionalmente, en la comparación obligada, se logra encontrar una gran diferencia técnica que es fundamental al evitar la extensa disección subcutánea requerida en las dos técnicas precedentes mencionadas por el comentarista, que ahora se reduce considerablemente en nuestra propuesta. La suspensión sobre la pared torácica para realzar y mantener el volumen glandular del polo superior de la misma, es ahora sin duda accesible, como lo mostramos evidentemente en el artículo.

El Dr. Vázquez, curiosamente le da poca importancia a lo que también proponemos acerca de la simplificación de la marcación cutánea. El reducir la longitud de la cicatriz final tradicionalmente establecida, resulta sin embargo de mucho beneficio para la mujer. La extensa T invertida, aunque técnicamente más fácil de ejecutar, no es ahora tan aceptable como alternativa resultante de cicatriz final. La suspensión a la pared torácica sin embargo, permite reducirla sin duda. Si se logra reducir la longitud de las incisiones, esto solo puede brindar agrado a las personas involucradas. Considero que un diagnóstico de hipertrofia mamaria no es sinónimo de cicatrices extensas como resultante. El reposicionar cefálicamente a voluntad un surco submamario, obviando las cicatrices acostumbradas, no deja de resultar atractivo y sirve para reducir la longitud final de las incisiones. Una "T" corta es entonces solo un sinónimo de bienestar, que puede resultar un buen cambio en lugar de lo acostumbrado. Si además, técnicamente lo facilita, invariablemente lo amerita. Agradezco los comentarios y la oportunidad de respuesta. 